\title{
EMC3-Eirene simulations of particle- and energy fluxes to main chamber- and divertor plasma facing components in ASDEX Upgrade compared to experiments
}

\author{
T. Lunt ${ }^{\mathrm{a}}$, D. Carralero ${ }^{\mathrm{a}}$, Y. Feng ${ }^{\mathrm{b}}$, G. Birkenmeier ${ }^{\mathrm{a}}$, H.W. Müller ${ }^{\mathrm{a}}$, S. Müller ${ }^{\mathrm{a}}$, M. \\ Wischmeier ${ }^{\mathrm{a}}$, the ASDEX Upgrade team ${ }^{\mathrm{a}}$ \\ ${ }^{a}$ Max-Planck-Institut für Plasmaphysik, Boltzmannstr. 2, 85748 Garching, Germany \\ ${ }^{b}$ Max-Planck-Institut für Plasmaphysik, Wendelsteinstr. 1, 17491 Greifswald, Germany
}

\begin{abstract}
We report on first EMC3-Eirene simulations with an extended computational grid including both divertor- and main chamber (MC) 3D wall plasma-facing components (PFCs). In a first step we compare the simulations to low-power L-mode discharges systematically analyzed by Carralero et al., who observed a transition from a low- to a high density regime [1]. Case A is a configuration at low density and high clearance, while $\mathrm{B}$ is a high density regime configuration at medium clearance. In order to explain the upstream far-SOL $n_{e}$ profiles of B the MC PFCs and an enhanced transport region at $\rho=1.01 \ldots 1.03$ need to be included in the simulations. In a second step we compute the particle-, and power fluxes to the limiter for the realistic geometry, for limiters displaced radially inward and for a toroidally symmetric limiter. Almost the same fraction of power as that to the divertor is absorbed by the MC PFCs in the simulation of case B.
\end{abstract}

Keywords:

PACS: 52.55.Rk, 52.40.Hf, 52.40.Fd, 52.30.Ex, 52.65.Pp

PSI-21:ASDEX Upgrade, EMC3, First wall, Detachment, Divertor modelling

Email address: tilmann.lunt@ipp.mpg.de (T. Lunt) 


\section{Introduction}

Currently one of the most promising designs for the realization of a fusion reactor is the divertor tokamak. In such a configuration a significant fraction of the power produced (or absorbed) in the confinement region will be guided to the strike zones on the divertor plates via parallel transport in the Scrape-off layer (SOL). Since the area of these zones constitutes only a tiny fraction of the total first wall surface, extremely high and strongly inhomogeneous power flux densities close to the material limits are found there. For this reason a reactor will most likely need to be operated in the 'detachment' regime, where the power- and particle fluxes to the divertor are strongly reduced and which is observed at high densities. At high densities Carralero et al. observed a transition to a regime with significantly flatter gradients in the SOL [1]. In particular the power fall-off length $\lambda_{q}$ increases by a factor of 4-5 during this 'high density transition' (HDT). For the same clearance (separatrix- to main chamber wall distance) the main chamber $(\mathrm{MC})$ components may then receive a significant fraction of the power $P_{\text {sep }}$ passing the separatrix. Given that the MC wall surface is by a factor of $\sim 100$ larger than that of the divertor strike zones, this might be an option to distribute the power over a larger area. However, electron- and ion temperatures in the range of several tens of eV [2] as well as charge exchange neutrals up to keV energies produced by recycling [3] are observed in AUG. The bombardment by these charged and non-charged particles can lead to large sputtering yields at the MC plasma-facing components (PFCs), a reduction of their life-time and possibly to a contamination of the confinement region (located closely to the sputtering region) by impurities. In fact MC impurity sources - although significantly smaller than those of the divertor - have been found to contribute most to the impurity content in the confined plasma [4]. Additionally, volume of the confined plasma is one of the most important optimization parameters, and so the clearance should not be too large. For the construction of a fusion reactor this raises the question: What is the optimum position of the MC plasma facing components (PFCs)? MC PFCs are often neglected in the simulations due to the technical difficulties contructing suitable computational grids. Nevertheless, such grids were successfully constructed recently e.g. for SOLPS $[5,6]$. The far-SOL region is often topologically 
O23 - Lunt et al.

more complicated due to the possible formation of a secondary separatrix, but also because MC PCFs, like limiters, ICRH-antenna, ECRH-mirrors or diagnostics are usually toroidally asymmetric and actually require a full 3D treatment of the transport.

For all these reasons the Edge Monte Carlo 3D-Eirene (EMC3-Eirene) code package [7] is particularly suitable for the inclusion of MC PFCs: The code solves the plasma fluid- and kinetic neutral equations in 3D on computational grids that firstly may intersect with the target and secondary do not necessarily need to be aligned with the flux surfaces. This second feature allows the construction of a topologically simple extension of the grid beyond the secondary separatrix.

In the following we report on the first EMC3-Eirene simulations including the AUG MC PFCs. For a detailed description of the code and its application the reader is referred to Refs. $[7,8,9]$. In Sec. 2 we compare the simulations to two low-power L-mode discharges systematically analyzed by Carralero et al. [1], one at low density and one after the HDT, in order to determine transport coefficients particularly in the far-SOL. Finally we scan the clearance by artificially moving the low-field side (LFS) limiters radially inward in Sec. 3 and summarize in Sec. 4.

\section{Simulations \& transport coefficients in the far-SOL}

A poloidal cross-section of a typical computational grid used for the simulations together with the AUG machine contours as well as the diagnostics referred to in the following is shown in Fig. 1. Radially the grid is devided into three parts, 'core' (red), 'SOL' (blue and magenta) and private flux region ('PFR', green). Due to the toroidal $180^{\circ}$ symmetry of the MC PFCs in AUG, the grid covers half of the circumference. The core region extends from $\rho_{p o l}=\sqrt{\Psi_{N}}=0.9$ to $\rho_{p o l}=1$ and the SOL from $\rho_{p o l}=1$ to $\rho_{p o l}>1.15$ with the radial surfaces of the grid being aligned to the flux surfaces up to $\rho_{p o l}=1.13$ (cf. blue part of the grid in Fig. 1) while the rest is a linear interpolation between the $\rho_{\text {pol }}=1.13$ surface and the vessel contour (cf. magenta colored part), which intersects with the MC PFCs and the second separatrix (cf. orange line) in a complicated way. The SOL grid is divided into $\sim 60 \times 600 \times 128$ cells in radial, poloidal and toroidal directions, respectively. Two auxiliary limiters at the 
toroidal angles $\phi=19^{\circ}, 64^{\circ}$ and two ICRH antenna with limiters on both sides, i.e. at $\phi=85^{\circ}, 108^{\circ}, 130^{\circ}, 154^{\circ}$ intersect the grid (these positions are shifted by $|\Delta \phi| \lesssim 1^{o}$ from the actual positions to align them symmetrically with the grid). The limiter leading edge is described by two circle slices with the radii $r_{1}=0.804 \mathrm{~m}$ and $r_{2}=1.034$ $\mathrm{m}$ and the central points at $(R, z)=\left(R_{0}-r_{i}, 0.119 \mathrm{~m}\right)$, where $R_{0}=2.205 \mathrm{~m}$ in the case of the ICRH antenna frames (cf. Fig. 1), while the auxiliary limiters (not shown) are located $5 \mathrm{~mm}$ further out at $R_{0}=2.210 \mathrm{~m}$.

The two cases analyzed are (A) discharge 29321 at 3.334 s, low density (edge line integrated density $\bar{n}_{e, \text { edge }}=2.2 \cdot 10^{19} \mathrm{~m}^{-3}$ ) and high clearance and (B) 29887 at 4.430 $\mathrm{s}$, high density $\left(\bar{n}_{e, e d g e}=3.5 \cdot 10^{19} \mathrm{~m}^{-3}\right)$ and medium clearance. The magnetic equilibria were computed by Cliste (1 ms time resolution) [10]. Both configurations are L-modes at rather low ECRH $P_{E C R H}=500 \mathrm{~kW}$ and ohmic $P_{O H}=500 \mathrm{~kW}$ heating power. Since we did not yet perform an impurity transport simulation, the impurity radiation of $P_{r a d}=500 \mathrm{~kW}$ measured by bolometry was subtracted from this, resulting in an input power of $P_{\text {in }}=500 \mathrm{~kW}$ equally distributed between electrons and ions. In the simulations the average density on the separatrix was used as a fixed boundary condition during the iteration process and adjusted such that the value $n_{e, M P}$ at the outboard midplane is $n_{e, M P}=1.0 \cdot 10^{19} \mathrm{~m}^{-3}$ in the case A and $n_{e, M P}=2.0 \cdot 10^{19} \mathrm{~m}^{-3}$ in the case $\mathrm{B}$ (note that $\bar{n}_{\text {sep }}$ is not necessarily equal to $n_{e, M P}$, see comments below).

The results of the simulation are shown in Fig. 2. The top plots show $n_{e}$ values upstream (blue curves) at the (3D) position of the Li-beam (blue data points) where they are compared to the local measurement. The green curve (simulation) and data points (measurements) show $n_{e}$ at $z=-0.965 \mathrm{~m}$, i.e. a few $\mathrm{cm}$ below the x-point, where the $\mathrm{X}$-point probe (XPR) is located. The ion saturation current densities $j_{\text {sat }}$ are shown in the bottom plots of Fig. 2, where the blue curves (simulation) and data points (measurements) correspond to the outer divertor, while red represents the inner one. The two plots in the middle show the particle cross-field diffusion coefficient $D_{\perp}$ and the connection length evaluated at the position of the Li-beam, which is strongly shortened by the inner heat shield and the auxiliary limiters shown by the gray vertical dashedand dotted lines, respectively. The ratio $\chi_{\perp} / D_{\perp}$, where $\chi_{\perp}$ is the heat cross-field diffusion coefficient for electrons and ions is kept constant to $\sim 7$. The solid lines show the 
O23 - Lunt et al.

case with MC PFCs, while the dashed lines show a reference simulation on the same grid, but without the MC PFCs.

In the case $A$ the simulations match those of the measurements upstream and at the target roughly within the error bars, when assuming $D_{\perp}=0.1 \mathrm{~m}^{2} / \mathrm{s}$ in the confinement region and $D_{\perp}=1.0 \mathrm{~m}^{2} / \mathrm{s}$ outside. Only at the XPR position the simulation overestimates the measurements, which may be due to the finite size of the probe and/or the locally induced particle recycling, effects not taken into account in the simulation. In the case $B$ the upstream profile matches that measured by the Li-beam for the case with MC PFCs (solid blue line), while it strongly deviates when the MC PFCs are removed in the simulation (dashed blue line), which shows the necessity to include these PFCs. In addition we needed to include a region ranging from $\rho=1.01$ to $\rho=1.03$ of enhanced transport $\left(D_{\perp}=2.5 \mathrm{~m}^{2} / \mathrm{s}\right)$ to achieve the flat gradient in that region. For comparison we also performed a simulation (dash-dotted curve) without this enhancement, i.e. with the same coefficients as in A, which has a significantly steeper slope than the experiment in the near-SOL region.

An effect observed at these high densities is a strong (factor $\sim 10$ ) peaking of the local density at the x-point, similar to that observed in EMC3-Eirene simulations for the snowflake divertor configuration in TCV [11]. The peaking of $n_{e}$ is accompanied by a drop in $T_{e}$ to values around $1 \mathrm{eV}$, where recombination is setting in, an effect not taken into account in the simulation. It is interesting to observe that the XPR in fact sees a strong decrease of $n_{e}$ at the x-point (green data points). Given that the recombination rate depends on $n_{e}^{2}$ one would expect that such a sink term limits the peaking of $n_{e}$ at the $\mathrm{X}$-point, however, it is hard to imagine that it actually causes a drop. It is even more unlikely that $n_{e}$ and $T_{e}$ drop simultaneously, which would require a large parallel pressure gradient on the flux surface as well as a heat removal mechanism. A possible explanation for the discrepancy could be that the deviation of the theoretical equilibrium from the experimental one is particularly large around the null point and that the low $n_{e}$ values actually correspond to the PFR. One might speculate that the production of (cold) neutrals at the x-point that are no longer bound to the field lines and can easily travel to the SOL regions (where they re-ionize or provoke charge exchange reactions removing momentum from the flowing plasma) plays a role in the detachment process 
observed at the inner divertor (Fig. 2 bottom right). Apart from recombination it might also be necessary to take into account the re-absobtion of photons in the x-point region plasma, which was found to become optically thick by Kotov et al. [12].

The power decay lengths for the two simulations are $\lambda_{q}=13 \mathrm{~mm}(\mathrm{~A})$ and $\lambda_{q}=22 \mathrm{~mm}$ (B). The latter is a mean value since the profile is not well described by an exponential. Experimentally a smaller $\lambda_{q}=5 \mathrm{~mm}$ is observed for case A in [1] while the value is matched well for case B.

\section{Scan of the limiter-separatrix distance}

With the same transport coefficients as in Sec. 2 we now compute the (electron and ion) particle- and power fluxes to all MC PFCs, while decreasing the clearance by moving the limiters radially inward (i.e. decreasing the minimum $\rho_{\text {lim }}$ value along the divertor contour). We will refer to the fraction of $P_{\text {in }}$ which is transferred to the neutrals via (ionic) charge exchange- and elastic collisions as well as electronic excitation processes as $P_{\text {neu }}$. $P_{\text {neu }}$ will also be deposited on a surface, but in a rather homogeneous way. The results are summarized in Tab. 1, where the configurations with the actual limiter positions are marked by the symbol $*$. We also compare the real geometry with $N_{\text {lim }}=6$ to an artificial toroidally symmetric limiter $\left(N_{\text {lim }}=1\right)$. While the power fluxes to the MC PFCs $P_{M C}$ in the case A are below $12 \%$ even with the limiters very close to the separatrix, $P_{M C}$ increases to $40 \%$ of $P_{i n}$ in the case B, i.e. to the same value as the fluxes to the divertor $P_{d i v}$. Also the particle fluxes to the MC PFCs $\Phi_{M C}$ are significant, although the effect is a little less pronounced as in the case of the power. This underlines the importance of the MC PFCs in the analysis in particular when studying the high density regime.

Finally we estimate the sputtering yields $Y\left(E_{0}\right)$ from Bohdansky's formula, which was revised by Eckstein [13] for tungsten as target material. The ions in front of the surface are accelerated in the sheath electric field, which affects them $Z$ times stronger than D. Including the energy the ions had when arriving the sheath edge, their final energy is often assumed to be $E_{i}=2 k_{B} T_{i}+3 k_{B} Z T_{e}$. Since the threshold energy $E_{t h}$ is of the order $200 \mathrm{eV}$ and $Z=1$ for deuterium, the background plasma is usually not predominantly 
responsible for the sputtering. For light impurities, like carbon or nitrogen with $E_{t h} \leq$ $50 \mathrm{eV}$ and $Z \sim 2 \ldots 3, E_{i}$ can more easily exceed $E_{t h}$. To estimate realistic sputtering yields one obviously needs to compute the light impurity transport in order to know their distribution, which is not done yet. Taking as a rough approximation that the $\mathrm{C}^{3+}$ flux $\Gamma_{C^{3+}}=c \Gamma_{D}$ is proportional to that of deuterium $\Gamma_{D}$, the total sputtering flux $\Phi_{W, M C}=\int_{M C} c \cdot \Gamma_{D} \cdot Y\left(E_{0}\right) d S$ increases by a factor of 4 , when passing from case A to case B. Although a W transport simulation is still missing it is likely that these sources also contribute more to the contamination of the confinement region than in case $\mathrm{A}$, stressing the importance to study this regime in view of ITER and DEMO.

\section{Summary and outlook}

For the first time particle- and power fluxes to main chamber- (MC) and divertor plasma facing components (PFCs) were simulated simultaneously in ASDEX Upgrade by EMC3-Eirene. Two configurations were compared (A) discharge 29321 at $3.334 \mathrm{~s}$ and high clearance and (B) 29887 at $4.430 \mathrm{~s}$ and medium clearance. Case A is below and $\mathrm{B}$ above the high density transition point observed by Carralero et al. The simulations match the upstream $n_{e}$ profiles of $\mathrm{A}$ and $\mathrm{B}$ measured by the Li-beam roughly within the error bars, when including the MC PFCs in both cases. In case $B$ it was also mandatory to increase the transport coefficients in a region $\rho=1.01 \ldots 1.03$ as shown by a reference simulation with the same coefficients as in A. The simulated downstream $j_{\text {sat }}$ profile matches that measured by the divertor probes in case $\mathrm{A}$, while the code generally overestimates the measurements of the $\mathrm{x}$-point probe in particular for case B at the x-point. With the transport coefficients used to fit the upstream profiles (electron and ion) particle- and energy fluxes were computed for A and B for different limiter positions. It was found that the same power as that going to the divertor is deposited on the MC PFCs in the case B underlining the importance of these PFCs for the power balance.

A more comprehensive study of configurations including discharges at high power is forseen for the near future, as well as a transport simulation of light impurities and of W sputtered from the MC PFCs. Furthermore, it would be instructive to include effects 
O23 - Lunt et al.

of recombination as well as photon transport in EMC3-Eirene to learn more about the physics at the x-point. 


\begin{tabular}{|c|c|c|c|c|c|c|c|}
\hline case & $\rho_{\text {lim }}$ & $N_{\text {lim }}$ & $\frac{P_{d i v}}{P_{\text {in }}}[\%]$ & $\frac{P_{M C}}{P_{\text {in }}}[\%]$ & $\frac{P_{\text {neu }}}{P_{\text {in }}}[\%]$ & $\frac{\Phi_{\text {div }}}{\Phi_{\text {rec }}}[\%]$ & $\frac{\Phi_{M C}}{\Phi_{\text {rec }}}[\%]$ \\
\hline $\mathrm{A}^{*}$ & 1.063 & 6 & 76.9 & 9.2 & 14.0 & 95.2 & 4.8 \\
$\mathrm{~A}$ & 1.042 & 6 & 76.0 & 10.8 & 13.5 & 93.7 & 6.3 \\
$\mathrm{~A}$ & 1.021 & 6 & 75.1 & 11.4 & 13.8 & 93.8 & 6.2 \\
$\mathrm{~A}$ & 1.021 & 1 & 74.9 & 11.7 & 13.7 & 93.9 & 6.1 \\
\hline $\mathrm{B}^{*}$ & 1.051 & 6 & 40.3 & 40.9 & 18.7 & 76.8 & 23.2 \\
$\mathrm{~B}$ & 1.040 & 6 & 41.2 & 43.5 & 15.8 & 74.8 & 25.2 \\
$\mathrm{~B}$ & 1.021 & 6 & 28.1 & 55.9 & 15.9 & 53.3 & 46.7 \\
$\mathrm{~B}$ & 1.021 & 1 & 32.4 & 54.7 & 13.8 & 67.8 & 32.2 \\
\hline
\end{tabular}

Table 1: Power- and particle fluxes $P$ and $\Phi$ fluxes to divertor- and MC PFCs, normalized to the input power $P_{i n}$ and the total recycling flux $\Phi_{r e c}$, respectively. $P_{n e u}$ is the power transferred to neutral particles via charge exchange and elastic collisions as well as electronic excitation processes. The inner heat shield is located at $\rho=1.054$ and $\rho=1.036$ for cases A and B, respectively. The experimental configurations marked by *. 
O23 - Lunt et al.

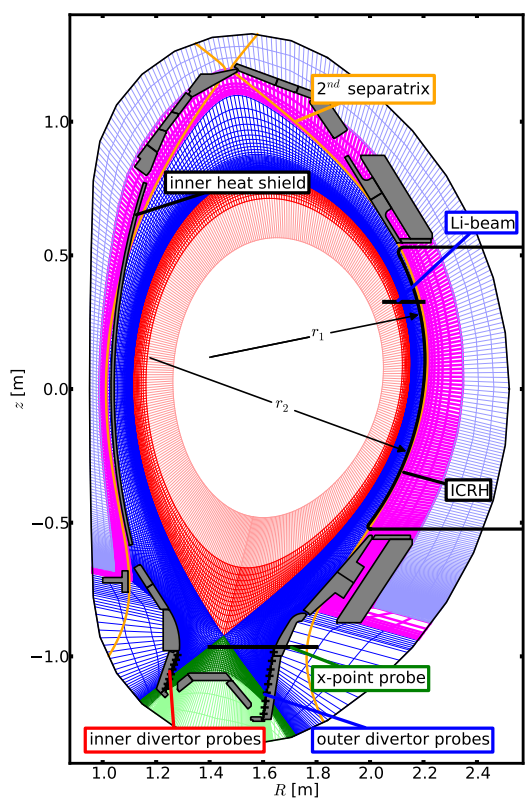

Figure 1: Poloidal cross section of the computational grid for EMC3-Eirene, AUG vessel structures and position of Li-beam, x-point manipulator- and divertor probes. 

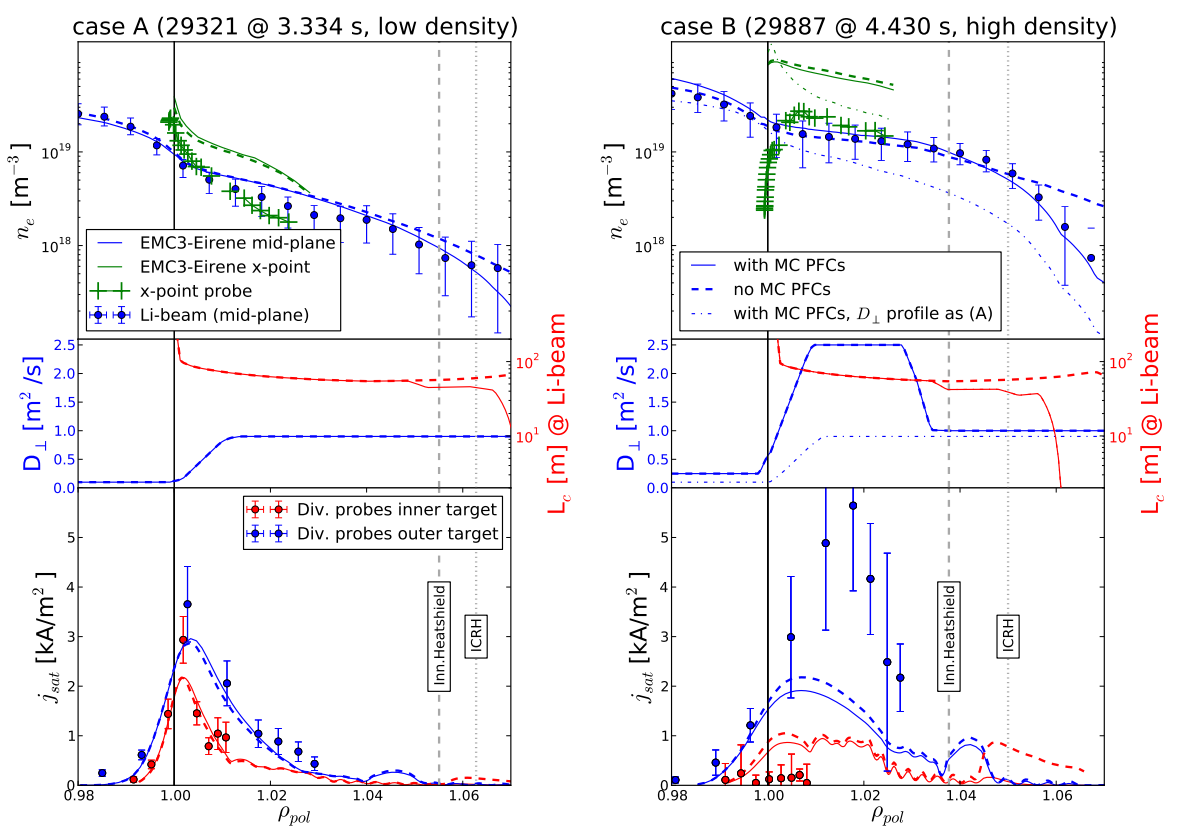

Figure 2: EMC3-Eirene simulation compared to Li-beam, x-point manipulator- and divertor probes. Left: case (A), low density and right: case (B) high density. The solid lines in both plots represent the case withand the dashed lines the case without MC PFCs. The dash-dotted curves in B correspond to a reference simulation with the same transport coefficients as in A with MC PFCs. 


\section{References}

[1] Carralero D. et al. Submitted to Nucl. Fusion (2014)

[2] Kocan M. et al. J. Nucl. Mater. 415 (2011) S1133-S1138

[3] Verbeek H. et al. Nucl. Fusion 38 (1997) 1789-1803

[4] Dux R. et al. J. Nucl. Mater. 390-391 (2009) 858-863

[5] Klingshirn H.J. et al. J. Nucl. Mater. 438 (2014) S856-S860

[6] Baelmans M. et al. Nucl. Fusion 51 (2011) 083023

[7] Feng Y. et al. Contrib. Plasma Phys. 44 (2004) No. 1-3, 57-69

[8] Lunt T. el al. Plasma Phys. Control. Fusion 53 (2011) 125010

[9] Lunt T. el al. Nucl. Fusion 52 (2012) 054013

[10] Mc Carthy P.J. Phys. of Plasmas 6 (1999) 3554

[11] Lunt T. el al. Plasma Phys. Control. Fusion 56 (2014) 035009

[12] Kotov V. el al. Plasma Phys. Control. Fusion 54 (2012) 082003

[13] Eckstein W. el al. Sputtering Data Max-Planck-Institut für Plasmaphysik Laboratory report 9/82 (1993) 